TRANSACTIONS OF THE

AMERICAN MATHEMATICAL SOCIETY

Volume 361, Number 7, July 2009, Pages 3707-3720

S 0002-9947(09)04687-X

Article electronically published on February 10, 2009

\title{
QUASI-ANOSOV DIFFEOMORPHISMS OF 3-MANIFOLDS
}

\author{
T. FISHER AND M. RODRIGUEZ HERTZ
}

\begin{abstract}
In 1969, Hirsch posed the following problem: given a diffeomorphism $f: N \rightarrow N$ and a compact invariant hyperbolic set $\Lambda$ of $f$, describe the topology of $\Lambda$ and the dynamics of $f$ restricted to $\Lambda$. We solve the problem where $\Lambda=M^{3}$ is a closed 3-manifold: if $M^{3}$ is orientable, then it is a connected sum of tori and handles; otherwise it is a connected sum of tori and handles quotiented by involutions.

The dynamics of the diffeomorphisms restricted to $M^{3}$, called quasi-Anosov diffeomorphisms, is also classified: it is the connected sum of DA-diffeomorphisms, quotiented by commuting involutions.
\end{abstract}

\section{INTRODUCTION}

This paper deals with hyperbolic sub-dynamics. It is related to a problem posed by M. Hirsch, around 1969: given a diffeomorphism $f: N \rightarrow N$ and a compact invariant hyperbolic set $\Lambda$ of $f$, describe the topology of $\Lambda$ and the dynamics of $f$ restricted to $\Lambda$. Hirsch asked, in particular, whether the fact that $\Lambda$ were a manifold $M$ would imply that the restriction of $f$ to $M$ is an Anosov diffeomorphism [11. However, in 1976, Franks and Robinson gave an example of non-Anosov hyperbolic sub-dynamics in the connected sum of two $\mathbb{T}^{3}[3$. (see below). There are also examples of hyperbolic sub-dynamics in non-orientable 3-manifolds; for instance, the example of Zhuzhoma and Medvedev [18. Here we show that all examples of 3 -manifolds that are hyperbolic invariant sets are, in fact, finite connected sums of the examples above and handles $S^{2} \times S^{1}$ (see definitions in $₫ 3$ and $\$ 5$ ).

Theorem 1.1. Let $f: N \rightarrow N$ be a diffeomorphism, and let $M \subset N$ be a hyperbolic invariant set for $f$ such that $M$ is a closed orientable 3 -manifold. Then the KneserMilnor prime decomposition of $M$ is

$$
M=T_{1} \# \ldots \# T_{k} \# H_{1} \# \ldots \# H_{r},
$$

the connected sum of $k \geq 1$ tori $T_{i}=\mathbb{T}^{3}$ and $r \geq 0$ handles $H_{j}=S^{2} \times S^{1}$. In case $M$ is non-orientable, then $M$ decomposes as

$$
M=\tilde{T}_{1} \# \ldots \# \tilde{T}_{k} \# H_{1} \# \ldots \# H_{r},
$$

the connected sum of $k \geq 1$ tori quotiented by involutions $\tilde{T}_{i}=\mathbb{T}^{3} \mid \theta_{i}$ and $r$ handles $H_{j}=S^{2} \times S^{1}$.

Received by the editors May 8, 2007.

2000 Mathematics Subject Classification. Primary 37D05, 37D20.

Key words and phrases. Dynamical systems, hyperbolic set, robustly expansive, quasi-Anosov.

This work was partially supported by NSF Grant \#DMS0240049, Fondo Clemente Estable 9021 and PDT.

(C)2009 American Mathematical Society Reverts to public domain 28 years from publication 
In 1976, Mañé obtained the following characterization [15] (see also Theorem 3.3) $: g: M \rightarrow M$ is the restriction of another diffeomorphism to a hyperbolic set $M$ that is a closed manifold, if and only if $g$ is quasi-Anosov. That is, if it satisfies Axiom A and all intersections of stable and unstable manifolds are quasi-transversal, i.e.,

$$
T_{x} W^{s}(x) \cap T_{x} W^{u}(x)=\{0\} \quad \forall x \in M .
$$

The Franks-Robinson example of a non-Anosov quasi-Anosov diffeomorphism is essentially as follows: they consider a hyperbolic linear automorphism of a torus $T_{1}$ with only one fixed point, and its inverse in another torus $T_{2}$. They produce appropriate deformations on each torus (DA-diffeomorphisms) around their respective fixed points. Then they cut suitable neighborhoods containing these fixed points, and carefully glue them together along their boundary so that the stable and unstable foliations intersect quasi-transversally. This is a quasi-Anosov diffeomorphism in the connected sum of $T_{1}$ and $T_{2}$, and hence $T_{1} \# T_{2}$ is a compact invariant hyperbolic set of some diffeomorphism. The non-orientable example by Medvedev and Zhuzhoma [18 is similar to Franks and Robinson's, but they perform a quotient of each $T_{i}$ by an involution before gluing them together.

The second part of this work, a classification of the dynamics of quasi-Anosov diffeomorphisms of 3-manifolds, shows that all examples are, in fact, connected sums of the basic examples above:

Theorem 1.2. Let $g: M \rightarrow M$ be a quasi-Anosov diffeomorphism of a closed 3-manifold $M$. Then:

(1) The non-wandering set $\Omega(g)$ of $g$ consists of a finite number of codimensionone expanding attractors, codimension-one shrinking repellers and hyperbolic periodic points.

(2) For each attractor $\Lambda$ in $\Omega(g)$, there exist a hyperbolic toral automorphism $A$ with stable index one, a finite set $Q$ of A-periodic points, and a linear involution $\theta$ of $\mathbb{T}^{3}$ fixing $Q$ such that the restriction of $g$ to its basin of attraction $W^{s}(\Lambda)$ is topologically conjugate to a DA-diffeomorphism $f_{Q}^{A}$ on the punctured torus $\mathbb{T}^{3}-Q$ quotiented by $\theta$. In case $M$ is an orientable manifold, $\theta$ is the identity map. An analogous result holds for the repellers of $\Omega(g)$.

Item (2) above is actually a consequence of item (11), as it was shown by Plykin in 20, 21]; see also [6] and [7. A statement of the result can be found in Theorem 4.3 in this work. The proof of Theorem 1.2 is in 84 . Theorem 1.2, in fact, implies Theorem 1.1. This is proved in $\$ 5$.

Let us see how a handle $S^{2} \times S^{1}$ could appear in the prime decomposition of $M$ : consider a linear automorphism of a torus $T_{1}$ and its inverse in a torus $T_{2}$, as in Franks-Robinson's example. Then, instead of exploding a fixed point, one explodes and cuts around an orbit of period 2 in $T_{1}$ and in $T_{2}$. The rest of the construction is very similar, gluing carefully as in that example to obtain quasi-Anosov dynamics. This gives the connected sum of two tori and a handle. The explanation and details can be found in $\$ 5$.

Let us also mention that in a previous work 22] it was shown that there exist a codimension-one expanding attractor and a codimension-one shrinking repeller if $g$ is a quasi-Anosov diffeomorphism of a 3-manifold that is not Anosov. The fact that only $\mathbb{T}^{3}$ can be an invariant subset of any known Anosov system was already 
shown by A. Zeghib 29]. In that case, the dynamics is Anosov. See also [2] and 16.

This work is also related to a work by Grines and Zhuzhoma [8]. There they prove that if an $n$-manifold supports a structurally stable diffeomorphism with a codimension-one expanding attractor, then it is homotopy equivalent to $\mathbb{T}^{n}$ and is homeomorphic to $\mathbb{T}^{n}$ if $n \neq 4$. In a certain sense, the results deal with complementary extreme situations in the Axiom A world: the Grines-Zhuzhoma result deals with structurally stable diffeomorphisms, which are Axiom A satisfying the strong transversality condition. This means that all $x, y$ in the non-wandering set satisfy at their points $z$ of intersection: $T_{z} W^{s}(x) \pitchfork T_{z} W^{u}(y)$. In particular,

$$
\operatorname{dim} E_{x}^{s}+\operatorname{dim} E_{y}^{u} \geq n \text {. }
$$

In our case, we deal with quasi-Anosov diffeomorphisms, which are Axiom A satisfying equality (1.1). In particular, for $x, y, z$ as above, we have $T_{z} W^{s}(x) \cap T_{z} W^{u}(y)=$ $\{0\}$, so

$$
\operatorname{dim} E_{x}^{s}+\operatorname{dim} E_{y}^{u} \leq n .
$$

In the intersection of both situations are, naturally, the Anosov diffeomorphisms.

Observe that it makes sense to get a classification of the dynamical behavior of quasi-Anosov on its non-wandering set, since quasi-Anosov are $\Omega$-stable [15] (see also \$3). They form an open set, due to quasi-transversality condition (1.1). Moreover, they are the $C^{1}$-interior of expansive diffeomorphisms; that is, they are robustly expansive [14. However, 3-dimensional quasi-Anosov diffeomorphisms of $M \neq \mathbb{T}^{3}$ are never structurally stable, so they are approximated by other quasiAnosov diffeomorphisms with different dynamical behavior, but similar asymptotic behavior (Proposition 3.2).

Finally, in Section 7 we study quasi-Anosov diffeomorphisms in the presence of partial hyperbolicity (see the definitions in \$7). We obtain the following result under mild assumptions on dynamical coherence:

Theorem 1.3. If $f: M^{3} \rightarrow M^{3}$ is a quasi-Anosov diffeomorphism that is partially hyperbolic, and either $E^{c s}$ or $E^{c u}$ integrate to a foliation, then $f$ is Anosov.

\section{BASIC DEFINITIONS}

Let us recall some basic definitions and facts: given a diffeomorphism $f: N \rightarrow N$, a compact invariant set $\Lambda$ is a hyperbolic set for $f$ if there is a $T f$-invariant splitting of $T N$ on $\Lambda$ :

$$
T_{x} N=E_{x}^{s} \oplus E_{x}^{u} \quad \forall x \in \Lambda,
$$

such that all unit vectors $v^{\sigma} \in E_{\Lambda}^{\sigma}$ with $\sigma=s, u$ satisfy

$$
\left|T f(x) v^{s}\right|<1<\left|T f(x) v^{u}\right|
$$

for some suitable Riemannian metric |.|. The non-wandering set of a diffeomorphism $g: M \rightarrow M$ is denoted by $\Omega(g)$ and consists of the points $x \in M$, such that for each neighborhood $U$ of $x$, the family $\left\{g^{n}(U)\right\}_{n \in \mathbb{Z}}$ is not pairwise disjoint. The diffeomorphism $g: M \rightarrow M$ satisfies Axiom $A$ if $\Omega(g)$ is a hyperbolic set for $g$ and periodic points are dense in $\Omega(g)$. The stable manifold of a point $x$ is the set

$$
W^{s}(x)=\left\{y \in M: d\left(f^{n}(x), f^{n}(y)\right) \rightarrow 0 \quad \text { if } \quad n \rightarrow \infty\right\},
$$

where $d(.,$.$) is the induced metric; the unstable manifold W^{u}(x)$ is defined analogously for $n \rightarrow-\infty$. If $g$ satisfies Axiom A, then $W^{s}(x)$ and $W^{u}(x)$ are immersed 
manifolds for each $x \in M$ (see for instance [25]). Also, if $d_{\sigma}$ is the intrinsic metric of the invariant manifold $W^{\sigma}(x)$, for $\sigma=s, u$, one has constants $C, \varepsilon>0$ and $0<\lambda<1$ such that, for instance, if $y \in W^{s}(x)$, and $d_{s}(x, y) \leq \varepsilon$ for some small $\varepsilon>0$, then

$$
d_{s}\left(f^{n}(x), f^{n}(y)\right) \leq C \lambda^{n} d_{s}(x, y) \quad \forall n \geq 0 ;
$$

an analogous bound holds for the unstable manifold.

Due to the Spectral Decomposition Theorem of Smale [26], if $g$ is Axiom A, then $\Omega(g)$ can be decomposed into disjoint compact invariant sets, called basic sets:

$$
\Omega(g)=\Lambda_{1} \cup \cdots \cup \Lambda_{r},
$$

each $\Lambda_{i}$ contains a dense orbit. Furthermore, each $\Lambda_{i}$ can be decomposed into disjoint compact sets $\Lambda_{i}=\Lambda_{i, 1} \cup \cdots \cup \Lambda_{i, k}$ such that there exists an $n \in \mathbb{N}$ where each $\Lambda_{i, j}$ is invariant and topologically mixing for $g^{n}$. A set $X$ is topologically mixing for a diffeomorphism $f$ if for each pair of non-empty open sets $U$ and $V$ of $X$, there is $K>0$ such that

$$
f^{k}(U) \cap V \neq \emptyset \quad \forall k \geq K
$$

Note that $\operatorname{dim} E_{x}^{s}$ is constant for $x$ varying on a basic set $\Lambda$. We shall call this amount the stable index of $\Lambda$ and will denote it by $\operatorname{st}(\Lambda)$.

For any set $\Lambda \subset M$, let us denote by $W^{\sigma}(\Lambda)$ the set $\bigcup_{x \in \Lambda} W^{\sigma}(x)$, where $\sigma=s, u$. We define the following (reflexive) relation among basic sets:

$$
\Lambda_{1} \rightarrow \Lambda_{2} \quad \Longleftrightarrow \quad W^{u}\left(\Lambda_{1}\right) \cap W^{s}\left(\Lambda_{2}\right) \neq \emptyset .
$$

The relation $\rightarrow$ naturally extends to a transitive relation $\succeq$ :

$$
\Lambda_{i} \succeq \Lambda_{j} \quad \Longleftrightarrow \quad \Lambda_{i} \rightarrow \Lambda_{k_{1}} \rightarrow \cdots \rightarrow \Lambda_{k_{r}} \rightarrow \Lambda_{j}
$$

where $\Lambda_{k_{1}}, \ldots, \Lambda_{k_{r}}$ is a finite sequence of basic sets. The diffeomorphism satisfies the no-cycles condition if $\succeq$ is anti-symmetric:

$$
\Lambda_{1} \succeq \Lambda_{2} \quad \text { and } \quad \Lambda_{2} \succeq \Lambda_{1} \quad \Longrightarrow \quad \Lambda_{1}=\Lambda_{2} .
$$

In this case $\succeq$ defines a partial order among basic sets.

We shall call $\Lambda$ an attractor if $\Lambda$ is a basic set such that $W^{u}(\Lambda)=\Lambda$. Note that this implies that there exists a neighborhood $U$ of $\Lambda$ such that $\Lambda=\bigcap_{n \in \mathbb{N}} f^{n}(U)$. Similarly, we shall call $\Lambda$ a repeller if $\Lambda$ is a basic set such that $W^{s}(\Lambda)=\Lambda$. If $g$ is Axiom A and satisfies the no-cycles condition, then hyperbolic attractors and repellers are, respectively, the minimal and maximal elements of $\succeq$.

A hyperbolic attractor $\Lambda$ is a codimension-one expanding attractor if all $x \in \Lambda$ satisfy $\operatorname{dim} W^{u}(x)=\operatorname{dim} M-1$. Codimension-one shrinking repellers are defined analogously.

Note that an attractor can have topological dimension $\operatorname{dim} M-1$ and still be not expanding. See the survey [9] on expanding attractors for a discussion on this topic.

\section{QUASI-ANOSOV DIFFEOMORPHISMS}

Let $f: N \rightarrow N$ be a diffeomorphism of a Riemannian manifold.

Definition 3.1. The sets $W^{s}(x)$ and $W^{u}(x)$ have a point of quasi-transversal intersection at $x$ if

(see Figure 1).

$$
T_{x} W^{s}(x) \cap T_{x} W^{u}(x)=\{0\}
$$




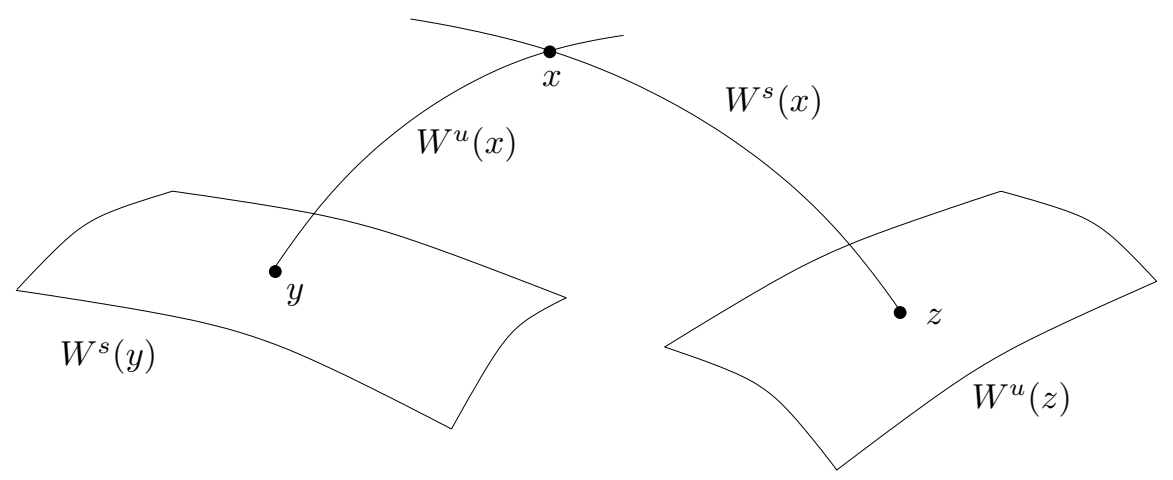

Figure 1. Quasi-transversal intersection at $x$

At a point of quasi-transversal intersection $x$, all vectors in $E_{x}^{s}$ form a positive angle with vectors in $E_{x}^{u}$. But this does not necessarily imply transversality, as can be seen in Figure 1 .

Let us note the difference between this definition and the strong transversality condition. There, transversality is required at the intersection points of $W^{u}(x)$ and $W^{s}(y)$, but this can be attained without quasi-transversality; for instance, if we had two planes intersecting at a curve in a 3-dimensional setting.

Observe that a structurally stable quasi-Anosov diffeomorphism is Anosov (see 15. and references therein). On the other hand, quasi-Anosov diffeomorphisms satisfy the no-cycles condition (see below), and hence they are $\Omega$-stable. Also, quasi-Anosov are a $C^{1}$-open set of diffeomorphisms [14.

Proposition 3.2. A quasi-Anosov diffeomorphism $f$ that is not Anosov is approximated by $\Omega$-conjugate quasi-Anosov diffeomorphisms that are not topologically conjugate to $f$.

The following theorem by Mañé relates quasi-Anosov diffeomorphisms with hyperbolic sub-dynamics.

Theorem 3.3 (Mañé [15]). A diffeomorphism $g$ is a quasi-Anosov diffeomorphism if and only if $M$ can be embedded as a hyperbolic set for a diffeomorphism $f: N \rightarrow$ $N$ by means of an embedding $i: M \hookrightarrow N$ satisfying $f i=i g$.

This characterization reduces the proof of Theorem 1.1 to proving Theorem 1.2. See also \$5. We shall review some properties of quasi-Anosov diffeomorphisms:

Proposition 3.4 ([15]). Quasi-Anosov diffeomorphisms satisfy the no-cycles condition.

Proof. If $\Lambda_{i}$ and $\Lambda_{j}$ are two basic sets satisfying $\Lambda_{i} \rightarrow \Lambda_{j}$, then $W^{u}\left(x_{i}\right) \cap W^{s}\left(x_{j}\right) \neq \emptyset$ for some $x_{k} \in \Lambda_{k}$. It follows from quasi-transversality that

$$
\left[n-\operatorname{st}\left(\Lambda_{i}\right)\right]+\operatorname{st}\left(\Lambda_{j}\right)=\operatorname{dim} E_{x_{i}}^{u}+\operatorname{dim} E_{x_{j}}^{s} \leq n,
$$

where $n$ is the dimension of $M$, hence $\operatorname{st}\left(\Lambda_{j}\right) \leq \operatorname{st}\left(\Lambda_{i}\right)$. We get by transitivity that

$$
\Lambda_{i} \succeq \Lambda_{j} \quad \Rightarrow \quad \operatorname{st}\left(\Lambda_{i}\right) \geq \operatorname{st}\left(\Lambda_{j}\right) .
$$

Suppose that,

$$
\Lambda_{1} \rightarrow \Lambda_{2} \rightarrow \cdots \rightarrow \Lambda_{k} \rightarrow \Lambda_{1}
$$


We have, in the first place, that $\operatorname{st}\left(\Lambda_{i}\right)=\operatorname{st}\left(\Lambda_{1}\right)$. Hence all intersections $x_{i} \in$ $W^{u}\left(\Lambda_{i}\right) \cap W^{s}\left(\Lambda_{i+1}\right)$ for $i=1, \ldots, k-1$, and $x_{k} \in W^{u}\left(\Lambda_{k}\right) \cap W^{s}\left(\Lambda_{1}\right)$ are transversal, since

$$
\left[n-\operatorname{st}\left(\Lambda_{i}\right)\right]+\operatorname{st}\left(\Lambda_{1}\right)=\operatorname{dim} E_{x_{i}}^{u}+\operatorname{dim} E_{x_{1}}^{s}=n .
$$

This implies that the $x_{i}$ 's belong to $\Omega(g)$; hence $\Lambda_{i}=\Lambda_{i}$ for all $i=1, \ldots, k$, and so $g$ satisfies the no-cycles condition.

In the particular case of a quasi-Anosov diffeomorphism of a 3-dimensional manifold, this implies that there can be only basic sets with stable index 2 or 1 and that basic sets with stable index one can only succeed basic sets with stable index one. We delay the proof of the next proposition until the next section.

Proposition 3.5. If $f$ is a quasi-Anosov diffeomorphism and $\Lambda_{0}$ is a codimensionone expanding attractor, and if $\Lambda$ is a basic set satisfying $\Lambda \succeq \Lambda_{0}$ with $\operatorname{st}(\Lambda)=1$, then $\Lambda=\Lambda_{0}$ or else $\Lambda$ is a periodic point.

Analogously, if $\Lambda_{0}$ is a codimension-one repeller and $\Lambda$ is a basic set satisfying $\Lambda_{0} \succeq \Lambda$ with $\operatorname{st}(\Lambda)=2$, then $\Lambda=\Lambda_{0}$ or $\Lambda$ is a periodic point. This implies:

Proposition 3.6. All attractors of a quasi-Anosov diffeomorphism of a 3-dimensional manifold are codimension-one expanding attractors unless the diffeomorphism is Anosov. An analogous statement holds for repellers.

Proof. Indeed, let $\Lambda_{R}$ be a repeller such that $\operatorname{st}\left(\Lambda_{R}\right)=1$ (hence, not codimensionone). There is a maximal chain of $\succeq$ containing $\Lambda_{R}$. Let $\Lambda_{A}$ be a minimal element of that chain. Then, due to (3.3) in the proof above, $\Lambda_{A}$ is a codimension-one expanding attractor. But then Proposition 3.5 implies that the repeller $\Lambda_{R}$ equals $\Lambda_{A}$, since $\Lambda_{A}$ cannot be a periodic point. Therefore $\Lambda_{R}=\Lambda_{A}=M$.

Note that Propositions 3.5 and 3.6 above prove item (1) of Theorem 1.2 . Item (2) of Theorem 1.2 follows from results in next section.

\section{CODIMENSION-ONE EXPANDING ATTRACTORS AND SHRINKING REPELlERS - Proof of TheOREM 1.2}

Before proving Proposition 3.5 we review properties of codimension-one expanding attractors. A codimension-one expanding attractor $\Lambda$ is orientable if the intersection index of $W^{s}(x) \cap W^{u}(y)$ is constant at all its intersection points, for $x, y \in \Lambda$. This notion was first introduced by Grines [4, 5. Let us also recall the following result by Zhuzhoma and Medvedev:

Theorem 4.1 (Medvedev-Zhuzhoma [18]). If $M$ is an orientable closed 3-manifold, then all codimension-one expanding attractors and shrinking repellers are orientable.

Derived from Anosov (or DA-) diffeomorphisms were introduced by Smale in [26] (see also 28]). They are certain deformations of hyperbolic automorphisms of the torus. We shall use the following definition [20]:

Corresponding to a hyperbolic toral automorphism $A$ with stable index one and a finite set $Q$ of $A$-periodic points, there is a diffeomorphism $f_{Q}^{A}: \mathbb{T}^{3} \rightarrow \mathbb{T}^{3}$ diffeotopic to $A$, such that $\Omega\left(f_{Q}^{A}\right)=\Lambda \cup Q$, where $\Lambda$ is a codimension-one expanding attractor and $Q$ is a finite set of $f_{Q}^{A}$-repelling periodic points. The stable manifolds of $f_{Q}^{A}$ coincide with the stable manifolds of $A$, except for a finite set of lines $\mathcal{L}_{Q}$. Each line $L \in \mathcal{L}_{Q}$ contains a point $q \in Q$. The component of $L-\Lambda$ containing $q$ is 
an interval whose endpoints $p^{ \pm}$are periodic boundary points of $\Lambda$. We call $f_{Q}^{A}$ a DA-diffeomorphism.

Plykin obtained models for connected codimension-one expanding attractors using DA-diffeomorphisms [20, 21]. See also $\S 8$ of [8]. We shall also use some of his intermediate results:

Theorem 4.2 (Plykin 20]). If $\Lambda$ is a connected orientable codimension-one expanding attractor of a diffeomorphism $g: M^{3} \rightarrow M^{3}$, then $W^{s}(\Lambda)$ has the homotopy type of $\mathbb{T}^{3}-Q$, where $Q$ is a finite set of points. There is a finite pointcompactification $\overline{W^{s}(\Lambda)}$ of $W^{s}(\Lambda)$ having the homotopy type of $\mathbb{T}^{3}$, and a homeomorphism $\bar{g}: \overline{W^{s}(\Lambda)} \rightarrow \overline{W^{s}(\Lambda)}$ extending $\left.g\right|_{W^{s}(\Lambda)}$ and admitting two $\bar{g}$-invariant fibrations that extend, respectively, the stable and unstable manifolds of $\Lambda$.

An analogous result holds for non-orientable attractors: there exists a twosheeted covering $\pi: \overline{W^{s}(\Lambda)} \rightarrow W^{s}(\Lambda)$ and a covering homeomorphism $\bar{g}: \overline{W^{s}(\Lambda)} \rightarrow$ $\overline{W^{s}(\Lambda)}$ that commutes with the involution $\theta: \overline{W^{s}(\Lambda)} \rightarrow \overline{W^{s}(\Lambda)}$ associated to $\pi$, such that $\overline{W^{s}(\Lambda)}$ has the homotopy type of $\mathbb{T}^{3}[21$.

Let us note that results above do not require that $\Lambda$ has a dense orbit.

Theorem 4.3 (Plykin [20, 21]). If $\Lambda$ is a connected orientable codimension-one expanding attractor of a diffeomorphism $g: M^{n} \rightarrow M^{n}$ having a dense unstable manifold, then there exist a hyperbolic toral automorphism A with stable index one and a finite set $Q$ of A-periodic points, such that $\left.g\right|_{W^{s}(\Lambda)}$ is topologically conjugate to the DA-diffeomorphism $\left.f_{Q}^{A}\right|_{\mathbb{T}^{n}-Q}$.

If $\Lambda$ is non-orientable, then there is a two-sheeted covering $\pi: \overline{W^{s}(\Lambda)} \rightarrow W^{s}(\Lambda)$ with an associate involution $\theta: \overline{W^{s}(\Lambda)} \rightarrow \overline{W^{s}(\Lambda)}$ and a covering homeomorphism $g: \overline{W^{s}(\Lambda)} \rightarrow \overline{W^{s}(\Lambda)}$ commuting with $\theta$ that is topologically conjugate to a DAdiffeomorphism $f_{Q}^{A}$ as described above.

Let us note that, in the case where the manifold $M$ is a torus, this result was obtained in [7]; see also [6] for the two-dimensional case.

Next, we state some of the results obtained in 8 and follow the general outline and notation.

Let $\Lambda$ be a codimension-one expanding attractor. We will assume for now that $\Lambda$ is orientable. (The non-orientable case will follow by taking a double cover and looking at the orientable case.) A point $p$ is a boundary point of a codimensionone expanding attractor $\Lambda$ if there exists a connected component of $W^{s}(p)-p$, denoted $W_{\emptyset}^{s}(p)$, not intersecting $\Lambda$. Boundary points for hyperbolic codimensionone expanding attractors are finite and periodic [20]. For $z \in \Lambda$ and given points $x, y \in W^{s}(z)$ we denote $(x, y)^{s}$ (respectively $[x, y]^{s}$ ) the open (closed) arc of $W^{s}(z)$ with endpoints $x$ and $y$. If $p$ is a boundary point of $\Lambda$ and $x \in W^{u}(p)-p$, then there is a unique $\operatorname{arc}(x, y)_{\emptyset}^{s}$ such that $(x, y)^{s} \cap \Lambda=\emptyset$ and $y \in \Lambda$. If $z \in W^{s}(\Lambda)-\Lambda$, then either $z \in(x, y)_{\emptyset}^{s}$ for some $x$ and $y$ elements of the unstable manifolds of boundary points, or $z \in W_{\emptyset}^{s}(p)$ for some boundary point $p \in \Lambda$.

The boundary points $p_{1}$ and $p_{2}$ are called associated if for each point $x \in W^{u}\left(p_{1}\right)$ there exists an $\operatorname{arc}(x, y)_{\emptyset}^{s}$ where $y \in W^{u}\left(p_{2}\right)$, and similarly for each point $y \in$ $W^{u}\left(p_{2}\right)$ there is an $\operatorname{arc}(x, y)_{\emptyset}^{s}$ where $x \in W^{u}\left(p_{1}\right)$. The boundary point $p_{1}$ is said to be paired if there exists a boundary point $p_{2}$ such that $p_{1}$ and $p_{2}$ are associated 1

\footnotetext{
${ }^{1}$ This concept also appears as 2-bunched in the bibliography. It is not to be confused with the concept of center bunching used for partially hyperbolic diffeomorphisms.
} 
Two associated boundary points always have the same period $m$. If $\operatorname{dim}(M) \geq 3$, then all boundary points are paired.

For associated periodic points $p_{1}$ and $p_{2}$ let

$$
\varphi_{p_{1}, p_{2}}:\left(W^{u}\left(p_{1}\right)-p_{1}\right) \cup\left(W^{u}\left(p_{2}\right)-p_{2}\right) \rightarrow\left(W^{u}\left(p_{1}\right)-p_{1}\right) \cup\left(W^{u}\left(p_{2}\right)-p_{2}\right)
$$

be defined by $\varphi_{p_{1}, p_{2}}(x)=y$ whenever $(x, y)_{\emptyset}^{s}$. The continuous dependence of stable and unstable manifolds implies that $\varphi_{p_{1}, p_{2}}$ is a homeomorphism. We may naturally extend $\varphi_{p_{1}, p_{2}}$ to be a homeomorphism of $W^{u}\left(p_{1}\right) \cup W^{u}\left(p_{2}\right)$ to itself by defining $\varphi_{p_{1}, p_{2}}\left(p_{1}\right)=p_{2}$ and $\varphi_{p_{1}, p_{2}}\left(p_{2}\right)=p_{1}$.

Fix $D_{p_{1}}$ a closed disk in $W^{u}\left(p_{1}\right)$ containing $p_{1}$ in the interior such that $D_{p_{1}} \subset$ $\operatorname{int}\left(f^{m}\left(D_{p_{1}}\right)\right)$. The boundary of $D_{p_{1}}$ is a circle denoted $S_{p_{1}}$. The circles $S_{p_{1}}$ and $f^{m}\left(S_{p_{1}}\right)$ bound an annulus contained in $W^{u}\left(p_{1}\right)$ denoted $A_{p_{1}}$.

Since $\varphi_{p_{1}, p_{2}}$ is a homeomorphism we can define

- a closed disk $D_{p_{2}}=\varphi_{p_{1}, p_{2}}\left(D_{p_{1}}\right)$ in $W^{u}\left(p_{2}\right)$,

- a circle $S_{p_{2}}=\varphi_{p_{1}, p_{2}}\left(S_{p_{1}}\right)$, and

- an annulus $A_{p_{2}}=\varphi_{p_{1}, p_{2}}\left(A_{p_{1}}\right)$.

The set

$$
C_{p_{1}, p_{2}}=\bigcup_{x \in S_{p_{1}}}\left(x, \varphi_{p_{1}, p_{2}}(x)\right)_{\emptyset}^{s}
$$

is called a connecting cylinder of $p_{1}$ and $p_{2}$, and is homeomorphic to the open 2cylinder $S^{1} \times(0,1)$. The set

$$
S_{p_{1}, p_{2}}=D_{p_{1}} \cup D_{p_{2}} \cup C_{p_{1}, p_{2}}
$$

is called a characteristic sphere for $p_{1}$ and $p_{2}$ and is homeomorphic to a sphere.

Define

$$
A_{p_{1}, p_{2}}=\bigcup_{x \in A_{p_{1}}}\left[x, \varphi_{p_{1}, p_{2}}(x)\right]_{\emptyset}^{s},
$$

which is homeomorphic to an annulus times an interval. Let

$$
D_{p_{1}, p_{2}}=\bigcup_{j \geq 0} f^{j m}\left(A_{p_{1}, p_{2}}\right)=\bigcup_{x \in W^{u}\left(p_{1}\right)-\operatorname{int} D_{p_{1}}}\left[x, \varphi_{p_{1}, p_{2}}(x)\right]_{\emptyset}^{s}
$$

and denote $\pi_{p_{1}}$ as the projection from $D_{p_{1}, p_{2}}$ to $W^{u}\left(p_{1}\right)-\operatorname{int}\left(D_{p_{1}}\right)$. Then the triple $\left(D_{p_{1}, p_{2}}, W^{u}\left(p_{1}\right)-\operatorname{int}\left(D_{p_{1}}\right), \pi_{p_{1}}\right)$ is a trivial fiber bundle with fiber the interval $[0,1]$.

The following is Corollary 3.1 in [8].

Lemma 4.4. Let $\Lambda_{0}$ be a codimension-one orientable expanding attractor and $p_{1}, p_{2}$ are associated boundary points on $\Lambda_{0}$. Suppose $\Lambda$ is another basic set of $M$ for $f$ with $\operatorname{st}(\Lambda)=1$. If there exists a point $z \in \Lambda$ such that $W^{u}(z) \cap D_{p_{1}, p_{2}} \neq \emptyset$, then $W^{u}(z)$ intersects $C_{p_{1}, p_{2}}$.

Theorem 6.1 in $[8]$ is similar to the following lemma.

Lemma 4.5. Suppose $f$ is a quasi-Anosov diffeomorphism of a closed 3-manifold $M$ and $\Lambda_{0}$ is an orientable codimension-one expanding attractor of $f$. Let $\Lambda \neq \Lambda_{0}$ be a basic set of $\operatorname{st}(\Lambda)=1$ such that $W^{u}(\Lambda) \cap D_{p_{1}, p_{2}} \neq \emptyset$. Let $C \subset D_{p_{1}, p_{2}} \cap W^{u}(z)$ be a component of the intersection of $D_{p_{1}, p_{2}} \cap W^{u}(z)$, where $z \in \Lambda$ is a periodic point. Then $W^{u}(z) \cap C_{p_{1}, p_{2}}=C \cap C_{p_{1}, p_{2}} \neq \emptyset$, and this intersection consists of a unique circle, $S$, that is isotopic to $S_{p_{1}}$ and $S_{p_{2}}$. 
We will provide an outline of the proof (see also [8]), since some of the details are needed in the proof of Proposition 3.5. The statement of Theorem 6.1 in [8] assumes that the diffeomorphism is structurally stable. Structurally stable diffeomorphisms have the strong transversality property which implies that for all $x \in \Lambda_{0}$ and $z \in \Lambda$,

$$
\begin{aligned}
& W^{s}(x) \cap W^{u}(z)=W^{s}(x) \pitchfork W^{u}(z) \text { and } \\
& W^{u}(x) \cap W^{s}(z)=W^{u}(x) \pitchfork W^{s}(z) .
\end{aligned}
$$

This is the property used in the proof in [8]. However, if $f$ is quasi-Anosov and $\Lambda_{0}$ and $\Lambda$ are codimension-one, then for all $x \in \Lambda_{0}$ and $z \in \Lambda$ property (4.4) holds by the quasi-transversal property of quasi-Anosov diffeomorphisms.

First, we use Lemma 4.4 to show there is a component. Next, we use transversality to show that every component $C \cap C_{p_{1}, p_{2}}$ is a circle, $S$, that is isotopic to $S_{p_{1}}$ and $S_{p_{2}}$. So in fact each component divides $C_{p_{1}, p_{2}}$ into two cylinders.

Next, let $B_{S} \subset W^{u}(z)$ be a minimal disk bounded by $S$. Since $B_{S}$ is minimal it follows that $B_{S} \cap D_{p_{1}, p_{2}}=\emptyset$. Then there are two possibilities:

(1) No $B_{S}$ contains $z$.

(2) Some $B_{S}$ contains $z$.

It is shown that case (1) cannot occur. For case (2) since $f\left(C_{p_{1}, p_{2}}\right) \subset D_{p_{1}, p_{2}}$ we know $f(S) \cap S=\emptyset$ and $S$ is inside $f(S)$ in $W^{u}(z)$. It then follows that $S$ and $f(S)$ bound a closed annulus in $W^{u}(z)$ which is a fundamental domain of $W^{u}(z)$ contained in $D_{p_{1}, p_{2}}$. Thus the intersection of $W^{u}(z)$ and $C_{p_{1}, p_{2}}$ is a unique circle.

Proof of Proposition 3.5. To simplify the argument we first assume that the attractors are orientable. Let us suppose that $\Lambda$ is a codimension-one basic set that is not a hyperbolic attractor. Since periodic points are dense in $\Lambda$ and $\Lambda_{0}$ we may assume there exist periodic points $x \in \Lambda$ and $x_{0} \in \Lambda_{0}$ such that $W^{s}\left(x_{0}\right) \cap W^{u}(x) \neq \emptyset$. Let $y \in W^{s}\left(x_{0}\right) \cap W^{u}(x)$. Then from the previous lemma $y \in\left(y_{1}, y_{2}\right)_{\emptyset}^{s}$ for $y_{1}$ and $y_{2}$ in the unstable manifolds of associated boundary points $p_{1}$ and $p_{2}$, respectively.

Let $S_{p_{1}, p_{2}}$ be a characteristic sphere for $p_{1}$ and $p_{2}$ such that $\left(y_{1}, y_{2}\right)_{\emptyset}^{s} \subset C_{p_{1}, p_{2}}$, so $C_{p_{1}, p_{2}} \cap W^{u}(x) \neq \emptyset$. From the previous lemma we know that $W^{u}(x) \cap D_{p_{1}, p_{2}}$ is a unique component $C$. Furthermore, there is a fundamental domain of $W^{u}(x)$ contained in $D_{p_{1}, p_{2}} \subset W^{s}\left(\Lambda_{0}\right)$. The invariance of $W^{s}(\Lambda)$ implies that $W^{u}(x)-x \subset$ $W^{s}\left(\Lambda_{0}\right)$. Hence, $\left(W^{u}(x)-x\right) \cap \Lambda=\emptyset$. Since $W^{u}(x) \cap W^{s}(x)$ is dense in a component of $\Lambda$ given by the Spectral Decomposition Theorem, we know that $\Lambda$ is trivial and consists of the orbit of $x$. In this way, the result follows for orientable attractors $\Lambda_{0}$.

We now suppose that $\Lambda$ is a codimension-one basic set and $\Lambda_{0}$ is a codimensionone non-orientable attractor where $\Lambda \rightarrow \Lambda_{0}$. This implies that $M$ is non-orientable from 21. Let $\bar{M}$ be an orientable manifold and $\pi: \bar{M} \rightarrow M$ is a (non-branched) double covering of $M$. Then there exists a diffeomorphism $\bar{f}$ of $\bar{M}$ that covers $f$. Furthermore, $\bar{M}$ contains a hyperbolic orientable codimension-one expanding attractor $\bar{\Lambda}_{0}$ such that $\bar{\Lambda}_{0} \subset \pi^{-1}\left(\Lambda_{0}\right)$. The result now follows from the previous argument by lifting $\Lambda$.

\section{Proof of Theorem 1.1}

Let us recall some basic definitions and results, which can be found in [19]. The connected sum of two 3-manifolds is obtained by removing the interior of a 3 -cell from each 3-manifold, and then matching the resulting boundaries, using an orientation reversing homeomorphism. The connected sum of $M$ and $M^{\prime}$ is denoted 
$M \# M^{\prime}$. In order to add a handle to a connected 3 -manifold $M$, one removes the interior of two disjoint 3-cells from $M$ and matches the resulting boundaries under an orientation reversing homeomorphism. If one adds a handle to $M$, one obtains a manifold isomorphic to $M \# S^{2} \times S^{1}$. Note that $M \# S^{3}=M$.

A manifold $M \neq S^{3}$ is prime if $M=M_{1} \# M_{2}$ implies $M_{1}=S^{3}$ or $M_{2}=S^{3}$. We have the following Unique Decomposition Theorem (see also [13]):

Theorem 5.1 (Milnor [19]). Every 3-manifold $M \neq S^{3}$ can be written as a finite connected sum,

$$
M=M_{1} \# \ldots \# M_{k},
$$

where each $M_{i}$ is prime, $i=1, \ldots, k$, and is unique up to order and isomorphisms.

Note that the handles $S^{2} \times S^{1}$ are prime manifolds. The torus $\mathbb{T}^{3}$ is also prime.

Now, let us prove Theorem [1.1, Let $M$ be a hyperbolic invariant set for a diffeomorphism $f$ such that $M$ is a 3-manifold. Then $f \mid M$ is a quasi-Anosov diffeomorphism [15. Let us first assume that $M$ is orientable. Theorem 4.1 implies that all attractors and repellers of $f \mid M$ are orientable. Then Theorems 4.2 and 4.3 imply that the basin of attraction/repulsion of each attractor/repeller is homeomorphic to a finitely punctured torus and also that $f$ restricted to each basin is topologically equivalent to a DA-diffeomorphism. Let us first consider the simplest case of a nonAnosov quasi-Anosov diffeomorphism: one with just one attractor and one repeller. Let us furthermore suppose that the basin of attraction of the attractor is homeomorphic to a torus minus one point. If one takes a ball centered at that (repelling) point and cuts the pre-image of that ball under the DA-diffeomorphism, one obtains a fundamental domain, which is homeomorphic, under the conjugacy, to a fundamental domain $D$ of $f \mid M$, met just once by the orbit of every point neither in the attractor nor in the repeller of $f \mid M$. By connectedness, the basin of repulsion of the repeller must also be homeomorphic to a torus minus one (attracting) point $A$, and $f$ restricted to this basin is also conjugated to another DA-diffeomorphism. The image of $D$ under this second conjugacy consists of two spheres $S^{3}$, each bounding a ball containing $A$ in its interior. So, the fact that $D$ is a fundamental domain implies that $M$ is the connected sum of two tori, just like in the Franks-Robinson example [3].

Let us now suppose that we have an attractor and a repeller, but that the basin of attraction is homeomorphic to a torus minus $k$ points, with $k \geq 2$. One can assume that the $k$ points are fixed under the DA-diffeomorphism by taking a sufficiently high iterate of the diffeomorphism. A connectedness argument shows that the basin of repulsion of the repeller is also homeomorphic to a torus minus $k$ points. Now, the previous procedure shows that $M$ is obtained by removing $k$ 3-cells from each torus and matching the resulting boundaries, using an orientation reversing homeomorphism. Observe that this implies that

$$
M=T_{1} \# T_{2} \# H_{1} \# \ldots \# H_{k-1}
$$

where $T_{1}, T_{2}$ are tori and the $H_{i}$ are handles $S^{2} \times S^{1}$. Indeed, instead of simultaneously removing the $k 3$-cells of each torus, one can only remove one 3 -cell from each torus and glue along their boundaries by $f$, which reverses orientation. In this way one obtains the connected sum of two tori. The rest of the procedure consists in repeating the operation $(k-1)$ times: cutting two disjoint 3-cells of this connected sum and matching the resulting boundaries by a reversing orientation homeomorphism. This is the same as adding a handle. 
Now, in fact there is nothing special in having just one attractor and one repeller. In case there are more attractors or repellers, one proceeds inductively as in the previous cases until one obtains a finite connected sum of tori and handles.

Let us also consider the case where the non-wandering set has a basic set $\Lambda$ that is a periodic orbit. We may assume $\operatorname{st}(\Lambda)=\operatorname{st}\left(\Lambda_{0}\right)$, and that $\Lambda \rightarrow \Lambda_{0}$, where $\Lambda_{0}$ is a connected codimension-one attractor. Let $p$ and $q$ be associated boundary periodic points and $P_{1}, \ldots, P_{k}$ be the set of all periodic points with $\operatorname{st}\left(P_{i}\right)=1$ and

$$
W^{u}\left(P_{i}\right) \cap \bigcup_{x \in W^{u}(p)-\{p\}}\left[x, \phi_{p, q}(x)\right]_{\emptyset}^{s} \neq \emptyset
$$

for all $1 \leq i \leq k$. Let $x \in W^{u}(p)-\{p\}$ and

$$
\theta_{x}:\left[x, \phi_{p, q}(x)\right]_{\emptyset}^{s} \rightarrow[0,1]
$$

be a homeomorphism. Denote $P_{i}^{x}$ as the point of intersection between $\left[x, \phi_{p, q}(x)\right]_{\emptyset}^{s}$ and $W^{u}\left(P_{i}\right)$. By reordering the points, if necessary, assume that $\theta_{x}\left(P_{i}^{x}\right)<\theta_{x}\left(P_{j}^{x}\right)$ for $i<j$. Then for any other $x^{\prime} \in W^{u}\left(x^{\prime}\right)$ and $\theta_{x^{\prime}}$ defined similarly we have $\theta_{x^{\prime}}\left(P_{i}^{x^{\prime}}\right)<\theta_{x^{\prime}}\left(P_{j}^{x^{\prime}}\right)$ for $i<j$, since $W^{u}\left(P_{i}\right)$ is codimension-one.

For the rest of the construction we assume, with no loss of generality, that $p, q, P_{1}, \ldots, P_{k}$ are all fixed points. Define the set

$$
D=\bigcup_{x \in W^{u}(p)-\{p\}}\left[x, \phi_{p, q}(x)\right]_{\emptyset}^{s} \cup W_{\emptyset}^{s}(p) \cup W_{\emptyset}^{s}(q) \cup W^{s}\left(P_{1}\right) \cup \cdots \cup W^{s}\left(P_{k}\right) .
$$

Following the construction in the proof of Theorem 1 of [20] we can extend the diffeomorphism $\left.f\right|_{D}$ to a homeomorphism $\bar{f}$ on the compactification $\bar{D}=D \cup \alpha_{1} \cup$ $\cdots \alpha_{k+1}$ where each $\alpha_{i}$ is a repelling fixed point for $\bar{f}$. Fix $\epsilon>0$ sufficiently small and let

$$
B=\bigcup_{x \in W_{\epsilon}^{u}(p)-\{p\}}\left[x, \phi_{p, q}(x)\right]_{\emptyset}^{s} \cup W_{\emptyset}^{s}(p) \cup W_{\emptyset}^{s}(q) \cup\left(\bigcup_{i=1}^{k} W^{s}\left(P_{i}\right)\right) \cup\left(\bigcup_{i=1}^{k+1} \alpha_{i}\right) .
$$

Let $B_{\epsilon}(0)$ be the ball of size $\epsilon$ centered at the origin in $\mathbb{R}^{n-1}$. Then we can define a homeomorphism $F: B_{\epsilon}(0) \times[0,1] \rightarrow B$ so that

$$
\begin{aligned}
& \text { - } F(0,0)=p, \\
& \text { - } F(0,1)=q, \\
& \text { - } F\left(0, \frac{2 i}{2 k+1}\right)=P_{i} \text { for } 1 \leq i \leq k, \\
& \text { - } F\left(0, \frac{2 i+1}{2 k+1}\right)=\alpha_{i+1} \text { for } 0 \leq i \leq k, \\
& \text { - } F(x, t) \in\left[F(x, 0), \phi_{p, q}(F(x, 0))\right]_{\emptyset}^{s} \text {, and } \\
& \text { - } F(x, 0) \in W_{\epsilon}^{u}(p) \text {. }
\end{aligned}
$$

Furthermore, we can extend the unstable manifolds of the points in $p \cup P_{1} \cup \cdots \cup P_{k}$ to a codimension-one fibration of $\bar{D}$ and extend the stable foliation to a fibration of $\bar{D}$ with one-dimensional fibers. Similar to Corollary 7.2 in [8], there exists a compact arc $a_{p q} \subset \bar{D}$ with no self-intersections such that

$$
a_{p q}=p \cup W_{\emptyset}^{s}(p) \cup \alpha_{1} \cup W^{s}\left(P_{1}\right) \cup \alpha_{2} \cup \cdots \cup P_{k} \cup \alpha_{k+1} \cup W_{\emptyset}^{s}(q) \cup q .
$$

Let $\mathcal{P}$ be the set of saddle periodic points of stable index one that intersect $W^{s}(\Lambda)$. Following the above construction we compactify $W^{s}(\Lambda) \cup W^{s}(\mathcal{P})$ to a set $\overline{W^{s}(\Lambda)}$ and extend the diffeomorphism $f$ on $W^{s}(\Lambda) \cup W^{s}(\mathcal{P})$ to a homeomorphism $\bar{f}$ of $\overline{W^{s}(\Lambda)}$. Here $\overline{W^{s}(\Lambda)}=W^{s}(\Lambda) \cup W^{s}(\mathcal{P}) \cup \mathcal{A}$, where $\mathcal{A}$ consists of a set of 
repelling periodic points for $\bar{f}$. The proof of Theorem 7.1 in 8 ] extends to $\overline{W^{s}(\Lambda)}$ to show that $\overline{W^{s}(\Lambda)}$ is homeomorphic to $\mathbb{T}^{3}$.

In the case where there is at least one non-orientable attractor or repeller, $M$ is non-orientable. Theorem 4.3 implies that the basin of attraction or repulsion of this attractor or repeller is homeomorphic to a torus quotiented by an involution minus $k$ points. $f$ is doubly covered by a DA-diffeomorphism in this set, and the procedure of removing cells and matching the corresponding boundaries follows as in the previous cases, whence one obtains that

$$
M=\tilde{T}_{1} \# \ldots \# \tilde{T}_{n} \# H_{1} \# \ldots \# H_{k},
$$

where the $\tilde{T}_{j}=\mathbb{T}^{3} \mid \theta_{j}$ are tori quotiented by involutions $\theta_{j}$ (possibly the identity) and the $H_{i}$ are handles.

\section{An EXAMPle of A QUASI-AnOSOV DIFFEOMORPHISM WITH A BASIC SET THAT IS A PERIODIC ORBIT}

Both the example by Franks-Robinson [3] and the example by MedvedevZhuzhoma [18 are quasi-Anosov diffeomorphisms whose non-wandering set consists exclusively of one codimension-one expanding attractor and one shrinking repeller.

Let us construct an example of a quasi-Anosov diffeomorphism with a basic set consisting of a periodic orbit.

Let $A$ be a linear hyperbolic diffeomorphism of a 3 -torus $T_{1}$ having at least one fixed point, and such that the stable dimension is 1 . Make a deformation around a fixed point in order to obtain a DA-diffeomorphism with a repelling fixed point and a codimension-one expanding attractor. See details in Section 4 . This new diffeomorphism $h$ preserves the original stable foliation. Now, make a new deformation, also preserving the original stable foliation, such that the repelling fixed point turns into a saddle, and two repelling fixed points appear on its stable manifold, locally separated by the unstable manifold of the saddle point.

As in the Franks-Robinson example, cut two 3-balls $B_{2}$ and $B_{3}$ containing, respectively, the repelling fixed points of $T_{1}$. Now, take two 3-tori $T_{2}$ and $T_{3}$, and consider the dynamics of $h^{-1}$ on each one of them. Cut two 3-balls $B_{2}^{\prime}$ and $B_{3}^{\prime}$, each containing the attracting fixed points of $T_{2}$ and $T_{3}$ respectively. Carefully glue $T_{1}$ and $T_{j}$ along the boundary of $B_{j}$ and $B_{j}^{\prime}, j=2,3$, by means of an orientation reversing homeomorphism as described in section 95 . One obtains an Axiom A diffeomorphism of a manifold which is the connected sum of $T_{1}, T_{2}$ and $T_{3}: T_{1} \# T_{2} \# T_{3}$. The non-wandering set of this diffeomorphism consists of one codimension-one expanding attractor, two codimension-one shrinking repellers, and a hyperbolic fixed point. Proceeding as in [3] one perturbs the diffeomorphism producing a twist in the regions where the surgery was performed. Since this perturbation is local, it does not affect the hyperbolic behavior of the non-wandering set. In this way one obtains an Axiom A diffeomorphism satisfying the quasi-transversality condition, and with the above mentioned non-wandering set.

\section{Partially hyperbolic QUaSi-Anosov DifFeOmorPhisms}

In this section, we study quasi-Anosov diffeomorphisms in the presence of partial hyperbolicity. A diffeomorphism $f$ is called partially hyperbolic if there exists an invariant splitting of the tangent bundle $T M=E^{s} \oplus E^{c} \oplus E^{u}$ such that all unit 
vectors $v^{\sigma} \in E_{x}^{\sigma}, \sigma=s, c, u$ satisfy

$$
\left|T f_{x} v^{s}\right|<\left|T f_{x} v^{c}\right|<\left|T f_{x} v^{u}\right| \text { and }\left|T f_{x} v^{s}\right|<1<\left|T f_{x} v^{u}\right| .
$$

It is a known fact that there are unique invariant foliations $\mathcal{W}^{s}$ and $\mathcal{W}^{u}$ that are everywhere tangent, respectively, to $E^{s}$ and $E^{u}$ (see, for instance, [12]). However, $E^{c}$ is not integrable in general. It is an open problem if it is integrable in the case $\operatorname{dim} E^{c}=1$. Here, we shall consider an a priori mild hypothesis: either $E^{c s}$ or $E^{c u}$ integrates to a (codimension-one) foliation.

Let us prove Theorem 1.3. Assume that $E^{c u}$ integrates to a foliation $\mathcal{F}$. We may suppose that the manifold $M$ is not the 3 -torus, for otherwise the result would be immediate. Then, $M$ is not irreducible (that is, there is an embedded sphere $S^{2}$ not bounding any 3 -dimensional ball). Indeed, irreducible manifolds are prime, and Theorem 1.1 implies that $M$ is not prime unless it is the 3 -torus. Now, Theorem C.2., p. 45 of Roussarie 24 implies that a codimension-one foliation $\mathcal{F}$ of a manifold that is not irreducible has a compact leaf. This compact leaf $T$ must be homeomorphic to a 2-torus, since the strong unstable foliation has no singularities and does not contain closed leaves.

Take $p \in M$ a periodic point in $M$, of period $k$, such that the stable manifold of $p$ hits $T$. This implies that the set of leaves $f^{k n}(T)$ ( $f$ preserves the foliation) accumulates in $p$. Now, Haefliger [10] says that the set of points lying in a compact leaf is compact. In particular $p \in M$, the accumulating point of compact leaves, must be in a compact leaf $T_{0}$. It follows that $T_{0}$ is $f^{k}$-invariant. Moreover, by the above argument, $T_{0}$ must be a 2 -torus. Now, the local stable manifolds of $T_{0}$ form an open set $U$ satisfying $f^{k}(\bar{U}) \subset U$. This implies that $T_{0}$ is a basic set that is an attractor, but this contradicts Theorem $1.2(2)$.

\section{ACKNOWLEDGMENTS}

We want to thank the referees for valuable comments, and Raúl Ures for suggestions. The second author is grateful to the Department of Mathematics of the University of Toronto, and especially to Mike Shub, for their kind hospitality.

\section{REFERENCES}

[1] J. Franks, Anosov diffeomorphisms, Global Analysis Proc. Symp. Pure Math. 14 AMS Providence, Rhode Island (1970) 61-93 MR0271990(42:6871)

[2] J. Franks, Invariant sets of hyperbolic toral automorphisms, Amer. J. Math. 99 (1977) 10891095 MR0482846 (58:2891)

[3] J. Franks, C. Robinson, A quasi-Anosov diffeomorphism that is not Anosov, Trans. AMS 223 (1976) 267-278 MR0423420(54:11399)

[4] V. Grines, The topological equivalence of one-dimensional basic sets of diffeomorphisms on two-dimensional manifolds, Uspehi Mat. Nauk 29, 6 (180) (1974) 163-164 MR0440624 (55:13498)

[5] V. Grines, The topological conjugacy of diffeomorphisms of a two-dimensional manifold on one-dimensional orientable basic sets I, Trans. Moscow Math. Soc. 32 (1975) 31-56 MR0418161 (54:6203)

[6] V. Grines, The topological conjugacy of diffeomorphisms of a two-dimensional manifold on one-dimensional orientable basic sets II, Trans. Moscow Math. Soc. 34 (1977) 243-252 MR0474417 (57:14057)

[7] V. Grines, E. Zhuzhoma, Topological classification of orientable attractors on an $n$ dimensional torus, Russian Math. Surveys 34 (1979) 163-164 MR.548425 (80k:58071)

[8] V. Grines, E. Zhuzhoma, On structurally stable diffeomorphisms with codimension one expanding attractors, Trans. AMS 357 (2005), no. 2, 617-667 MR2095625 (2005k:37034) 
[9] V. Grines, E. Zhuzhoma, Expanding attractors, Regular and chaotic dynamics 11, 2 (2006) 225-246 MR2245079 (2007c:37015)

[10] A. Haefliger, Variétés feuilletés, Topologia Differenziale (Centro Int. Mat. Estivo, 1 deg Ciclo, Urbino (1962)). Lezione 2 Edizioni Cremonese, Rome 367-397 (1962) MR0163327 (29:630)

[11] M. Hirsch, On invariant subsets of hyperbolic sets, Springer, New York (1970) 126-135 MR0264684(41:9275)

[12] M. Hirsch, C. Pugh, M. Shub, Invariant manifolds, Lecture Notes in Math. 583 (1977) MR0501173 (58:18595)

[13] H. Kneser, Geschlossen Flächen in dreidimensionalen Mannigfaltigkeiten, Jahresbericht der Deutschen Mathematiker Vereinigung 38 (1929) 248-260

[14] R. Mañé, Expansive diffeomorphisms, Dyn. Syst., Proc. Symp. Univ. Warwick 1973/74, Lect. Notes Math. 468 (1975) 162-174. MR0650658 (58:31263)

[15] R. Mañé, Quasi-Anosov diffeomorphisms and hyperbolic manifolds, Trans. AMS 229 (1977) 351-370. MR 0482849 (58:2894)

[16] R. Mañé, Invariant sets of Anosov diffeomorphisms, Invent. Math. 46 (1978), 147-152 MR0488167 (58:7730)

[17] R. Mañé, A proof of the $C^{1}$ stability conjecture, Publ. Math. Inst. Hautes Étud. Sci. 66 (1988) 161-210 MR932138 (89e:58090)

[18] V. Medvedev, E. Zhuzhoma, On nonorientable two-dimensional basic sets on 3-manifolds, $S b$. Math. 193 (2002), no. 5-6, 869-888 MR.1957954(2004k:37040)

[19] J. Milnor, A unique decomposition theorem for 3-manifolds, Amer. J. of Math. 84, 1 (1962) 1-7 MR0142125 (25:5518)

[20] R. Plykin, Hyperbolic attractors of diffeomorphisms, Russian Math. Surveys 35, 3 (1980) 109-121. MR580625 (82k:58081)

[21] R. Plykin, Hyperbolic attractors of diffeomorphisms (the non-orientable case), Russian Math. Surveys 35, 4 (1980) 186-187. MR0586207 (82k:58082)

[22] J. Rodriguez Hertz, R. Ures, J. Vieitez, On manifolds supporting quasi-Anosov diffeomorphisms, C.R. Acad. Sci. 334, 4, 321-323 (2002) MR1891011(2002m:37040)

[23] C. Robinson, Dynamical Systems Stability, Symbolic Dynamics, and Chaos, CRC Press, 1999. MR.1792240 (2001k:37003)

[24] R. Roussarie, Sur les feuilletages des variétés de dimension trois, Ann. Inst. Fourier 21, 3 (1971) 13-82. MR.0348768 (50:1263)

[25] M. Shub Global stability of dynamical systems, with the collab. of A. Fathi and R. Langevin. Transl. by J. Cristy, New York, Springer-Verlag. XII (1987). MR.869255 (87m:58086)

[26] S. Smale, Differentiable dynamical systems, Bull. AMS, 73, 6 (1967) 747-817. MR0228014 $(37: 3598)$

[27] S. Smale, The $\Omega$-stability theorem, Global Analysis, Proc. Symp. Pure Math. 14 (1970) 289-297 MR0271971 (42:6852)

[28] R. Williams. The 'DA' maps of Smale and structural stability, Global Anal., Proc. Symp. Pure. Math., AMS 14 (1970), 329-334 MR0264705 (41:9296)

[29] A. Zeghib, Subsystems of Anosov systems, Amer. J. Math. 117 (1995), 1431-1448 MR1363074 (97e:58175)

Department of Mathematics, Brigham Young University, 292 tmCB, Provo, Utah 84602

E-mail address: tfisher@math.byu.edu

imerl, Facultad de Ingeniería, University de la Republica, Julio Herrera y Reissig 565, 11300 Montevideo, Uruguay

E-mail address: jana@fing.edu.uy 